\title{
Rotura Uterina às 18 Semanas de Gravidez no Contexto de Útero Malformado
}

\section{Uterine Rupture at 18 Weeks of Pregnancy in the Context of Malformed Uterus}

\author{
Emídio VALE-FERNANDES ${ }^{1}{ }^{1}$, Neusa TEIXEIRA ${ }^{1}$, Alexandra CADILHE ${ }^{1}$, Maria José ROCHA ${ }^{1}$ \\ Acta Med Port 2016 Oct;29(10):667-670 - http://dx.doi.org/10.20344/amp.7150
}

\section{RESUMO}

As malformações congénitas do tracto genital feminino são relativamente comuns e frequentemente assintomáticas. Apesar do resultado da gravidez poder ser favorável, estão descritos desfechos obstétricos adversos em mulheres com malformações uterinas. Os autores apresentam o caso clínico de uma emergência obstétrica, que realça a possibilidade de um desfecho muito adverso e raro, de rotura uterina da hemicavidade esquerda num útero bicórneo longe do termo, às 18 semanas de gestação, numa grávida com antecedentes de cesariana na hemicavidade direita e com placenta increta. Um útero malformado, com cavidade de tipo rudimentar apresenta menor distensibilidade da parede com a progressão da gravidez e facilita o desenvolvimento de formas anormais de placentação, aumentando o risco de rotura uterina no primeiro e segundo trimestres. O conhecimento da existência de uma malformação uterina no período pré-concepcional reveste-se de importância fundamental.

Palavras-chave: Canais de Müller; Desfecho Obstétrico; Placenta Acreta; Rotura Uterina; Útero/anomalias congénitas.

\section{ABSTRACT}

Birth defects of the female genital tract are relatively common and often asymptomatic. Despite the pregnancy outcome can be favorable, adverse obstetric outcomes are described in women with uterine malformations. The authors report the case of an obstetric emergency which enhances the possibility of a very adverse and rare outcome of uterine rupture in a left hemi-cavity of a bicornuate uterus away from the term, at 18 weeks of pregnancy, in a pregnant woman with history of caesarean in the right hemi-cavity and with placenta increta. A malformed uterus with a primitive type cavity has lower distensibility of the wall with the progression of the pregnancy and facilitates the development of abnormal placentation forms, increasing the risk of uterine rupture in the first and second trimesters. The knowledge of the existence of a congenital uterine anomaly in the preconceptional period is of primary importance.

Keywords: Mullerian Ducts; Placenta Accreta; Pregnancy Outcome; Uterine Rupture; Uterus/abnormalities.

\section{INTRODUÇÃO}

As malformações congénitas do tracto genital feminino (anomalias dos canais de Müller) são relativamente comuns ( $4 \%$ - $7 \%$ da população) e frequentemente assintomáticas, ${ }^{1}$ podendo atingir prevalências superiores em grupos seleccionados, nomeadamente mulheres com infertilidade ou com abortamentos de repetição. ${ }^{1,2}$

Existem vários sistemas de classificação e categorização das anomalias dos canais Müllerianos, destacando-se o publicado pela Sociedade Americana de Fertilidade em $1988,{ }^{3}$ pela sua ampla aceitação e utilização. Em 2012, a Sociedade Europeia de Reprodução Humana e Embriologia e a Sociedade Europeia de Endoscopia Ginecológica propuseram um novo sistema de classificação clínica baseado nas variações anatómicas em relação ao útero normal, levando em conta a sua origem embriológica. ${ }^{4,5} \mathrm{O}$ útero bicórneo resulta da fusão parcial dos canais Müllerianos (colo único e duas hemicavidades com graus variáveis de separação), e constitui uma malformação de tipo IV na classificação americana, e de classe três, de acordo com a categorização europeia referida. ${ }^{1-5}$

Apesar do resultado da gravidez poder ser favorável, estão descritos desfechos obstétricos adversos em mulheres com malformações uterinas (parto pré-termo, abortamentos de repetição e risco aumentado de parto por cesa- riana por apresentação fetal anómala). ${ }^{2}$ Os autores apresentam um caso clínico que realça a possibilidade de um resultado obstétrico muito adverso e raro de rotura uterina num útero bicórneo longe do termo da gravidez.

\section{CASO CLÍNICO}

Grávida de 38 anos, previamente saudável, com antecedentes de uma cesariana há sete anos por apresentação pélvica em trabalho de parto (gravidez de 37 semanas, de evolução normal, com recém-nascido com $2850 \mathrm{~g}$ ), admitida na sala de emergência, às 18 semanas de gravidez, por lipotimia, hipotensão e taquicardia. Ao exame objectivo apresentava abdómen inocente e ao exame ginecológico apresentava colo posterior, formado e fechado, sem sinais de hemorragia vaginal.

A ecografia obstétrica sumária realizada revelou morte fetal (feto com biometrias para 18 semanas) e um aparente nódulo miomatoso da parede uterina posterior, sem evidência de líquido livre na cavidade abdómino-pélvica. Analiticamente, a doente apresentava uma hemoglobina de $8,5 \mathrm{~g} / \mathrm{dL}$ e um hematócrito de $25,4 \%$, alterações sugestivas de anemia por hemorragia. Durante a observação, a doente apresentou uma dor abdominal súbita e de elevada intensidade. Dados os achados clínicos e laboratoriais

1. Serviço de Ginecologia e Obstetrícia. Hospital de Braga. Braga. Portugal.

$\triangle$ Autor correspondente: Emídio Vale-Fernandes. emidio.vale.fernandes@gmail.com

Recebido: 03 de novembro de 2015 - Aceite: 24 de maio de 2016 | Copyright @ Ordem dos Médicos 2016 


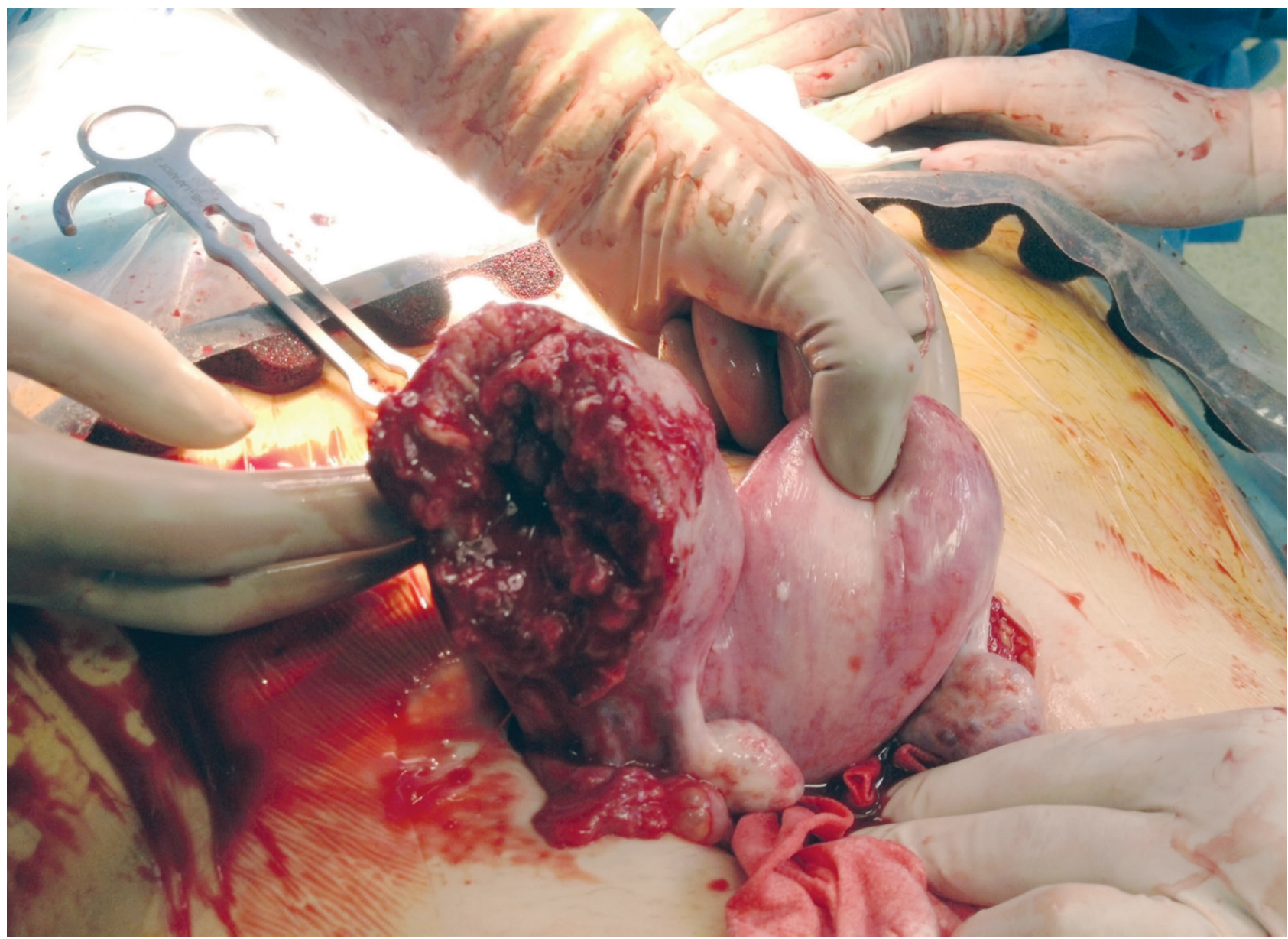

Figura 1 - Útero bicórneo com rotura lateral extensa da parede uterina da hemicavidade esquerda. De acordo com o sistema de classificação do grupo CONUTA - CONgenital UTerine Anomalies - da Sociedade Europeia de Reprodução Humana e Embriologia e da Sociedade Europeia de Endoscopia Ginecológica, a anomalia do tracto genital desta doente foi classificada como U3a, C0, V0 (Útero bicorpóreo (U3) com defeito de fusão parcial (a), Colo uterino normal (C0), Vagina normal (V0)). ${ }^{5} \mathrm{~A}$ imagem ecográfica pré-operatória sugestiva de nódulo miomatoso da parede posterior correspondia a um hematoma volumoso localizado na região da rotura uterina.

sugestivos de choque hipovolémico foi realizada uma laparotomia emergente. Durante a cirurgia constatou-se a existência de feto na cavidade peritoneal e um hemoperitoneu de grande volume.

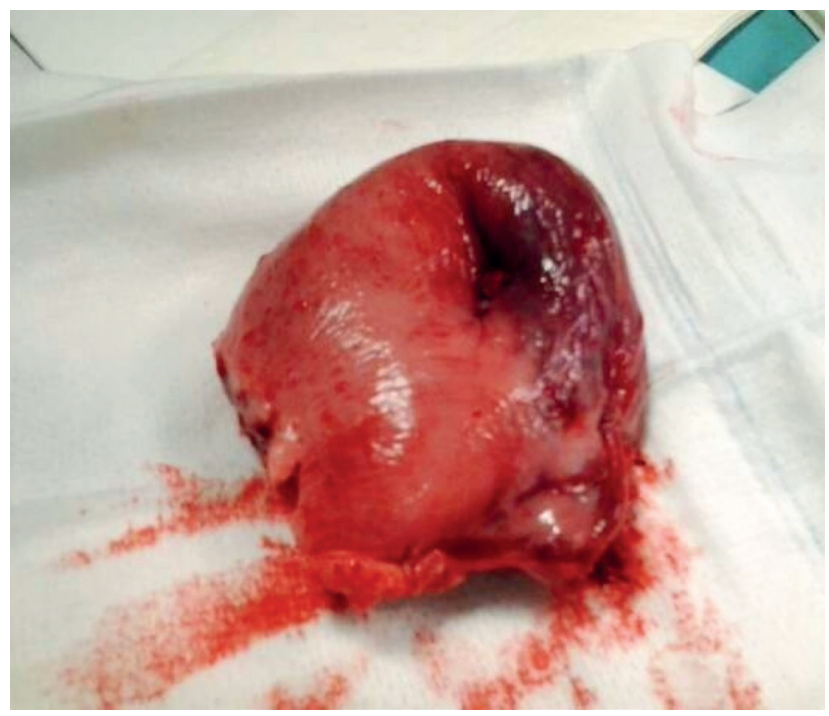

Figura 2 - Hemicavidade uterina esquerda removida
Diagnosticou-se intra-operatoriamente um útero bicórneo com uma grande rotura longitudinal na parede lateral da hemicavidade uterina esquerda (Fig. 1). A cicatriz da cesariana anterior foi localizada ao nível da hemicavidade uterina direita, sem relação com a região de rotura. Realizou-se a exérese cirúrgica de toda a hemicavidade uterina, por dificuldade na hemostase do local de inserção placentária, com conservação do anexo uterino ipsilateral (Fig.s 2 a 4). O controlo analítico no pós-operatório imediato revelou um valor de hemoglobina de $5,2 \mathrm{~g} / \mathrm{dL}$, com necessidade de transfusão de concentrados de eritrócitos. O pós-operatório decorreu sem intercorrências (alta hospitalar ao quinto dia pós-operatório).

O exame histológico da peça operatória confirmou uma hemicavidade uterina com miométrio normal com uma rotura longitudinal de oito centímetros e vilosidades coriónicas invadindo o miométrio (placenta increta). $O$ feto não apresentava alterações no hábito externo (Fig. 5). Foi realizada uma ecografia renal que excluiu anomalias concomitantes do aparelho urinário (presentes em $20 \%$ $30 \%$ das mulheres com anomalias dos canais de Müller). ${ }^{1}$ A doente foi aconselhada e alertada sobre as possíveis 


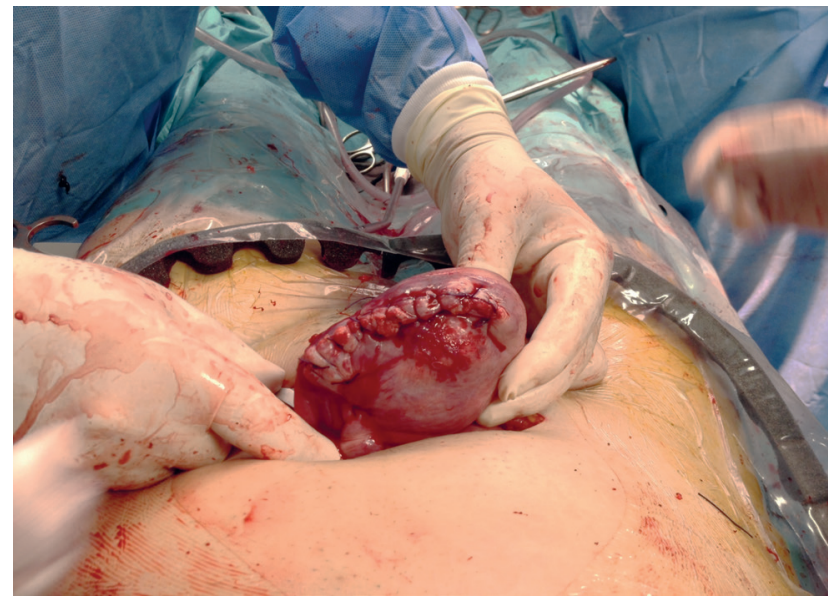

Figura 3 - Contorno lateral esquerdo do útero, após exérese cirúrgica da hemicavidade esquerda rota

implicações de uma futura gravidez, nomeadamente do risco de rotura uterina. Na consulta de revisão pós-operatória (após quatro semanas), a doente apresentava-se assintomática, sem alterações ao exame objectivo e manifestou vontade de não voltar a engravidar (iniciou contracepção hormonal oral combinada).

\section{DISCUSSÃO}

A rotura uterina na gravidez é uma complicação rara associada a elevada morbilidade e mortalidade materno-fetais, ocorrendo quase que exclusivamente no termo ou durante o trabalho de parto (incidência de $0,03 \%$ dos nascimentos). ${ }^{6} \mathrm{Na}$ grande maioria dos casos existem antecedentes de cesariana, sendo a incidência neste população de cerca de $0,1 \%{ }^{6}$ São também factores de risco a grande multiparidade, a utilização de ocitocina ou prostaglandinas na indução/aceleração do trabalho de parto, o parto auxiliado com fórceps, a placenta anormalmente aderente, as manobras de versão cefálica externa, a macrossomia fetal, a obesidade materna, a desproporção feto-pélvica, a apresentação fetal anómala, os antecedentes de cirurgia uterina, esvaziamento uterino ou histeroscopia e as malformações uterinas. ${ }^{6}$ Está descrita uma prevalência de $8 \%$ de roturas uterinas em mulheres com malformações uterinas que tentaram um parto vaginal após cesariana, comparativamente a um risco de $0,61 \%$ encontrado no grupo controlo. ${ }^{7}$

Um útero malformado apresenta uma menor distensibilidade. A parede uterina pode ser anormal ou tornar-se muito fina com o evoluir da gestação, aumentando o risco de rotura uterina. ${ }^{8,9}$ Além disso, está descrito um risco acrescido de desenvolvimento de formas anormais de placentação, que podem per si condicionar uma fraqueza adicional à parede uterina, em úteros malformados com cavidades de tipo rudimentar. ${ }^{8,10}$ No presente caso clínico, a gravidez desenvolveu-se na hemicavidade esquerda de um útero bicórneo, que rompeu às 18 semanas de gravidez, e documentou-se a existência de placenta increta (invasão do miométrio por vilosidades coriónicas sem atingimento da serosa uterina). A história anterior de parto por cesariana, após uma gravidez sem intercorrências na hemica-

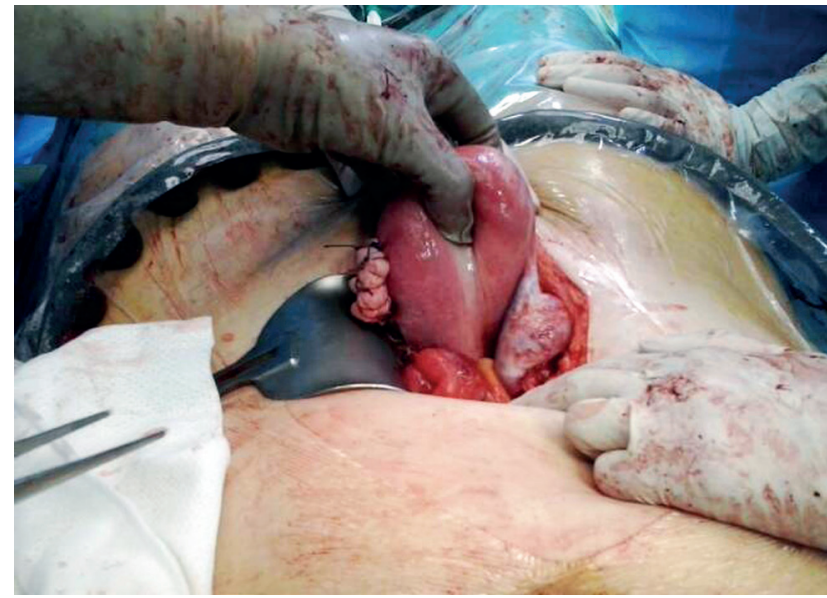

Figura 4 - Útero reconstruído, após exérese cirúrgica da hemicavidade esquerda rota, com conservação do anexo uterino ipsilateral

vidade direita, sem relação com a rotura uterina descrita, com recém-nascido com peso superior a $2500 \mathrm{~g}$, aliada ao facto da hemicavidade esquerda ter rompido numa fase precoce da gestação e na presença de placenta increta, poderá indicar que esta hemicavidade é de tipo rudimentar. A incidência de gravidez numa hemicavidade uterina de tipo rudimentar é de 1:40 000.8,9 Nestas situações o risco de rotura uterina tende a acontecer no primeiro ou segundo trimestres de gravidez, longe do termo da gestação. ${ }^{8-10}$ Estão descritos na literatura casos de rotura uterina em úteros com cavidades de tipo rudimentar desde as cinco semanas de gravidez. ${ }^{8}$

O diagnóstico de rotura uterina durante a gravidez exige um elevado grau de suspeição, constitui uma emergência obstétrica, pode estar associada a hemorragia catastrófica e é um desafio clínico, particularmente numa fase precoce da gestação. Os sinais e sintomas mais sugestivos de rotura uterina (no termo da gravidez) são a dor contínua no

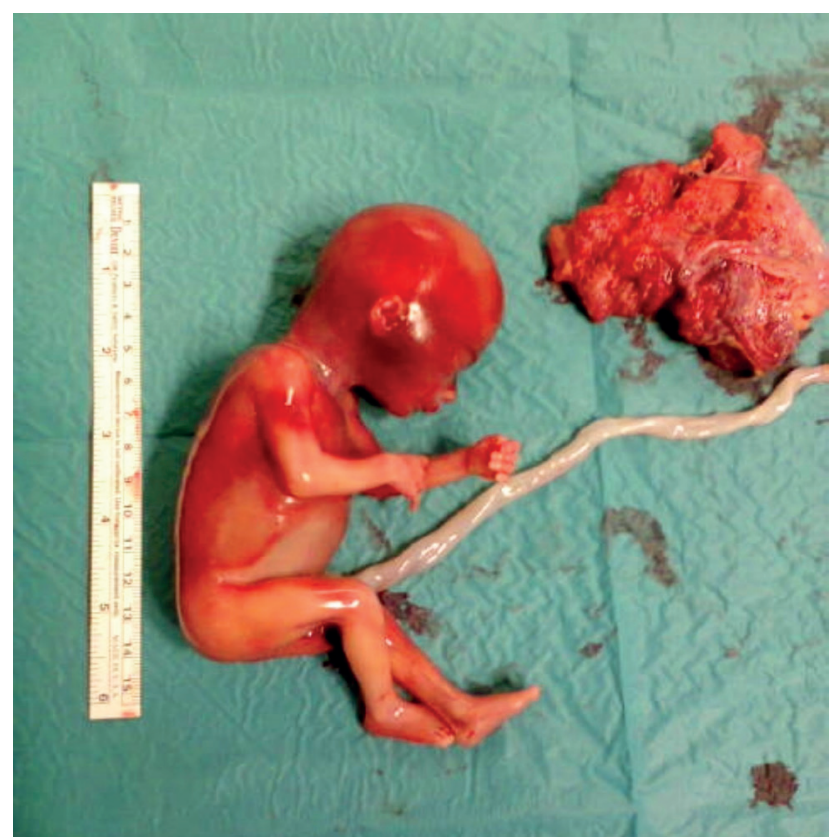

Figura 5 - Feto (biometrias para 18 semanas de gravidez e sem alterações aparentes do hábito externo), cordão umbilical e placenta 
local da cicatriz de cesariana, a hemorragia vaginal e a desaceleração prolongada da frequência cardíaca fetal (hipóxia fetal aguda). ${ }^{6}$ Nos casos de hemorragia abundante, como no presente caso de rotura uterina, com hemoperitoneu de grande volume, o choque hipovolémico constitui a forma de apresentação. O conhecimento da existência de uma malformação uterina no período pré-concepcional facilitará a abordagem diagnóstica da rotura uterina longe do termo da gravidez.

\section{OBSERVAÇÕES}

Este trabalho foi apresentado sob a forma de comunicação oral na $2^{\mathrm{a}}$ Reunião de Casos Clínicos em Ginecologia, sob a coordenação científica do Serviço de Ginecologia do Centro Hospitalar de Lisboa Norte - Hospital de Santa Maria e o patrocínio da Merck Sharp \& Dohme, e será publicado no Livro de Resumos do evento (31 de outubro de 2015, Hotel Dolce Campo Real).

\section{REFERÊNCIAS}

1. Chan YY, Jayaprakasan K, Zamora J, Thornton JG, Raine-Fenning N, Coomarasamy A. The prevalence of congenital uterine anomalies in unselected and high-risk populations: a systematic review. Hum Reprod Update. 2011;17:761-71.

2. Chan YY, Jayarpakasan K, Tan A, Thornton JG, Coomarasamy A, Raine-Fenning NJ. Reproductive outcomes in women with congenital uterine anomalies: a systematic review. Ultrasound Obstet Gynecol. 2011; 38:371-82.

3. American Fertility Society. The American Fertility Society classifications of adnexal adhesions, distal tubal occlusion, tubal occlusion secondary to tubal ligation, tubal pregnancies, Müllerian anomalies and intrauterine adhesions. Fertil Steril. 1988;49:944-55.

4. Grimbizis GF, Campo R, Gordts S, Brucker S, Gergolet M, Tanos V, et al. Clinical approach for the classification of congenital uterine malformations. Gynecol Surg. 2012;9:119-29.

5. Grimbizis GF, Gordts S, Di Spiezio SA, Brucker S, De Angelis C, Gergolet M, et al. The ESHRE-ESGE consensus on the classification of

\section{PROTECÇÃO DE PESSOAS E ANIMAIS}

Os autores declaram que os procedimentos seguidos estavam de acordo com os regulamentos estabelecidos pelos responsáveis da Comissão de Investigação Clínica e Ética e de acordo com a Declaração de Helsínquia da Associação Médica Mundial.

\section{CONFIDENCIALIDADE DOS DADOS}

Os autores declaram ter seguido os protocolos do seu centro de trabalho acerca da publicação de dados.

\section{CONFLITOS DE INTERESSE}

Os autores declaram a inexistência de conflitos de interesse na realização do presente trabalho.

\section{FONTES DE FINANCIAMENTO}

Os autores declaram que o presente trabalho não foi objecto de qualquer financiamento.

female genital tract congenital anomalies. Gynecol Surg. 2013;10:199212

6. Ayres-de-Campos D. Hipóxia fetal aguda. In: Ayres-de-Campos D Santos Silva I, Costa FJ. Emergências Obstétricas. Lisboa: Lidel; 2011. p. 9-27.

7. Ravasia DJ, Brain PH, Pollard JK. Incidence of uterine rupture among women with müllerian duct anomalies who attempt vaginal birth after cesarean delivery. Am J Obstet Gynecol. 1999;181:877-81.

8. Jayaprakash S, Muralidhar L, Sampathkumar G, Sexsena R. Rupture of bicornuate uterus. BMJ Case Rep. 2011; 2011.

9. Kore S, Pandole A, Akolekar R, Vaidya N, Ambiye VR. Rupture of left horn of bicornuate uterus at twenty weeks of gestation. J Postgrad Med. 2000;46:39-40.

10. Ashelby L, Toll G, Patel RR, Abdel-Fattah S, Hunter A. Live birth after rupture of a non-communicating horn of a bicornuate uterus. BJOG. 2005;112:1576-7. 